\title{
WAFER-LEVEL INTEGRATION OF NITI SHAPE MEMORY ALLOY WIRES FOR THE FABRICATION OF MICROACTUATORS USING STANDARD WIRE BONDING TECHNOLOGY
}

\author{
A. C. Fischer, H. Gradin, S. Braun, S. Schröder, G. Stemme and F. Niklaus \\ KTH - Royal Institute of Technology, Stockholm, Sweden
}

\begin{abstract}
This paper reports on the first integration of SMA wires into silicon based MEMS structures using a standard wire bonder. This approach allows fast and efficient placement, alignment and mechanical attachment of NiTi-based SMA wires to silicon-based MEMS. The wires are mechanically anchored and clamped into deep-etched silicon structures on a wafer. The placement precision is high with an average deviation of $4 \mu \mathrm{m}$ and the mechanical clamping is strong, allowing successful actuation of the SMA wires.
\end{abstract}

\section{INTRODUCTION}

Shape memory alloy (SMA) is an attractive actuator material to use in microelectromechanical systems (MEMS) when high forces and work are needed. SMA outperforms most other actuation principles at the microscale by more than an order of magnitude because of the high work density that SMAs offer [1]. Traditionally there are mainly two ways of integrating SMA materials into microsystems. The first approach is a pick and place approach where the SMA material and the microsystem is manufactured separately and then combined in a subsequent step [2]. This approach has the advantage of allowing integration of bulk SMA materials, which are commercially available and offered in a wide thickness range at comparably low material cost. However, the SMA integration is performed on a per-device level which results in high assembly cost. The second approach is based on sputter deposition of thin NiTi films directly onto the microstructure [3], which has the benefit of allowing wafer level processing. However, due to a difficult deposition controllability, the process is limited in reproducibility of transformation temperatures and strains and NiTi sputter deposition is mostly feasible for thicknesses of less than $10 \mu \mathrm{m}$ [4].

Wafer level integration of SMA wires onto silicon microstuctures, with the benefit of both wafer level integration and the use of bulk SMA materials, has been shown for microactuators with excellent perfomance [5]. However, no standardized fabrication process has yet been established or suggested. The placement of the wires requires specially designed tools with manual wire handling, alignment and integration. In contrast, wire bonding is an extremely mature, cost-effective and broadly available backend process for electrical interconnects [6]. It is very attractive to utilize this standard technology due to its very good availability and high performance in terms of reliability, throughput and placement accuracy, with speeds up to 22 bonds per second and placement accuracies within $2 \mu \mathrm{m}$. However, direct wire bonding of NiTi SMA wires is not feasible due to the Vickers hard- ness of the NiTi material, which is one order of magnitude higher [7] as compared to common wire bonding materials such as gold and aluminum. This paper presents the first wafer-level integration concept for NiTi SMA wires onto silicon substrates by using a conventional wire bonding tool.

\section{CONCEPT}

Wafer level integration of SMA wires with a wire bonder allows a fast placement and fixation of the wires. No additional alignment step is needed. Because of the hardness of NiTi wires the direct wire bonding of these wires on standard gold or aluminum pads is not feasible. In this paper the wires are instead mechanically fixed by Si clamping and anchor structures on the wafer. These mechanical fixation structures enable the integration of the NiTi wires by using a standard and unmodified wirebonder.

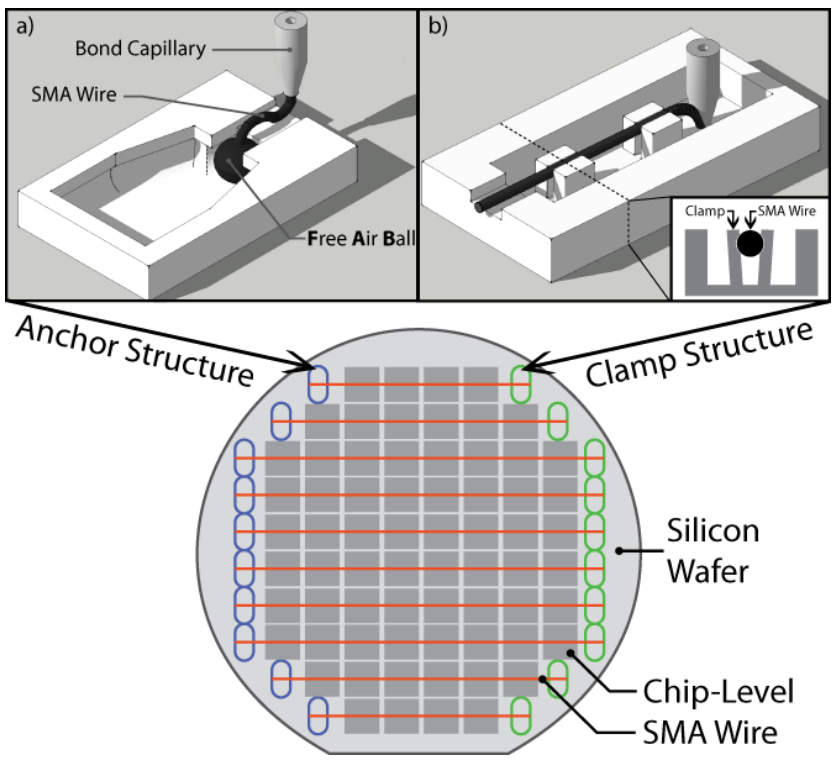

Figure 1: Illustration of the wafer-level integration concept: The SMA wire is mechanically fixed with the help of one anchor and one clamp structure. Each fixation pair is placed on opposite sides of the wafer edge.

The basic integration concept is depicted in Fig. 1. The integration of a SMA wire is separated into wafer- and chiplevel integration. Wafer-level integration combines aligning and fixation of a SMA wire on a silicon wafer. The final mechanical and electrical integration can then be performed on chip-level to form the actuator itself. 
(a)

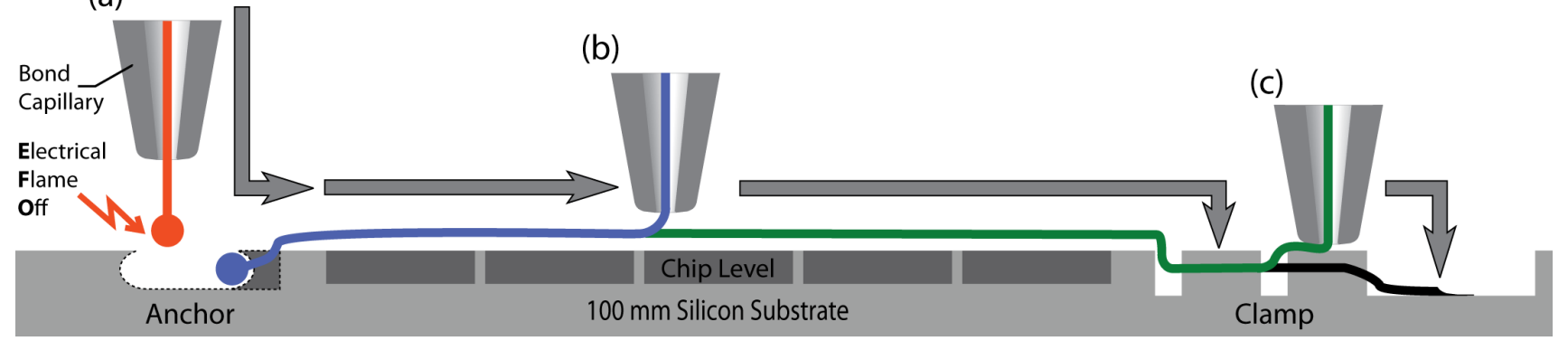

Figure 2: Cross sectional view of the integration flow: First, the free air ball is generated by an electrical discharge (a). The ball is then anchored in its anchoring structure. The SMA wire (37.5 $\mu \mathrm{m}$ diameter) is feeded and guided over the entire wafer area to its second fixation structure (b). The SMA wire is clamped in between silicon cantilevers and finally cut off by truncating the wire by the bond capillary and a high bond force (c).

At wafer-level, the SMA wire is attached using two different mechanical structures in a defined position with high placement accuracy across the entire wafer. Based on a ballwedge wire bonding process, a free air ball is generated and subsequently anchored in the first integration structure (Fig. 1a). The SMA wire is fixed and can be guided across the wafer towards the clamping structure (Fig. 1b), where the SMA wire is mechanically attached. This wafer-level integration allows in a second step a further attachment also on chip-level, e.g. by local clamping structures. However, the focus of this paper lies on the design and development of the wafer-level integration.

Using this integration concept, full wafers can be populated with SMA wires at high placement accuracies. Furthermore using a wire bonder offers high throughput and high reproducability of the integration process. The entire integration of SMA wires is done at room temperature, thus avoiding the triggering of the SMA transformation, which occurs at elevated temperatures of typically $90{ }^{\circ} \mathrm{C}$. Hence this enables integration of prestrained SMA wires. In addition this integration concept is CMOS compatible, offering the possibility for integrating microactuators with microelectronic devices.

\section{FABRICATION}

The wire hooking and clamping structures were fabricated on a $100 \mathrm{~mm}$ silicon substrate. Both types of structures are realized by a sequence of anisotropic and isotropic DRIE steps [8]. Etching of both integration structures were performed in a STS ICP etch reactor.

The fabrication of both fixation structures requires one lithography and DRIE process each. The first lithography was performed with standard photoresist, which was applied by spin-coating. The second photoresist application was performed by spray-coating of diluted resist in order to ensure a full protection of the deep-etched structures, which were created by the first DRIE process. As a final step all photoresist residuals were removed by plasma ashing.

The anchor structure, which fixes the start of the wire, is depicted in Fig. 1a and Fig. 3. It consists of two functional parts, the landing zone and the anchor part. The landing zone enables lowering of the SMA ball into the anchor structure and subsequently fixation into the adjacent anchor part. The etch geometry is crucial for a proper anchoring of the SMA ball. The anchoring of the SMA ball is achieved by a specific undercut etch profile of the anchor structure itself. First an anisotropic trench was etched, followed by a passivation of the vertical sidewalls. Subsequently an isotropic etch creates an undercut underneath the anisotropic etch profile.

The silicon structure for the wire end combines two functional parts, namely the clamping structure and the cut off zone. Fig. 1b), 2c) and 4 depict the clamping structure with a pair of facing vertical cantilevers. Two pairs of facing vertical cantilevers clamp the SMA wire in between them. The first pair fixes and aligns the SMA wire. The second pair stabilizes the SMA wire for the truncation. The anisotropic etch profile of the clamping structure contains a small chamfer on the upper section of the cantilevers, which is realized by a short isotropic etch. The chamfers ensure a constant height of the SMA wire in the clamp, as depicted in Fig. 4.

A semi-automatic wire-bonder (Delvotec 5410) was used to integrate the wires. A commercially available pre-strained NiTi SMA wire (Flexinol ${ }^{\circledR}, 37.5 \mu \mathrm{m}$ in diameter) with a transformation temperature of $90^{\circ} \mathrm{C}$ was used. Because the wire was already presrained, no special tool for prestraining the wires was needed [5]. The wire integration, depicted in Fig. 2, starts with forming a free air ball caused by an electrical discharge, which locally melts the wire on the tip of the bond capillary. The liquid metal at the end of the SMA wire rolls up and forms a free air ball. The Electrical Flame Off (EFO) is performed in helium atmosphere, since the TiNi alloys tend to oxidize in air during rapid melting by glow discharges [10]. The free air ball is then lowered into the landing zone and subsequently hooked into its adjacent anchoring part. By utilizing the wire bonder, the SMA wire is placed over the wafer to the clamping structure where it is pushed in between pairs of facing cantilevers. Finally, the SMA wire is cut off by truncating the wire with the bond capillary by a high bond force. This integration approach for SMA wires 
opens a wide range of different integration methods on chiplevel, which can then be used for manufacturing of high performance actuators with for example polymer fixation [5], or by using the clamp structures to fixate SMA wires also on individual chips.

\section{EXPERIMENTAL RESULTS}

Fig. 3 depicts an anchor structure, that was fabricated by combined anisotropic and isotropic deep reactive ion etching in three subsequent steps. Both the underetched (out of plane) sidewall structure and the tapered (in-plane) design safely fixes the SMA wire with its anchor. This design is very forgiving towards unequally formed and/or sized free air balls and thus ensures a reliable anchoring of the SMA wire.

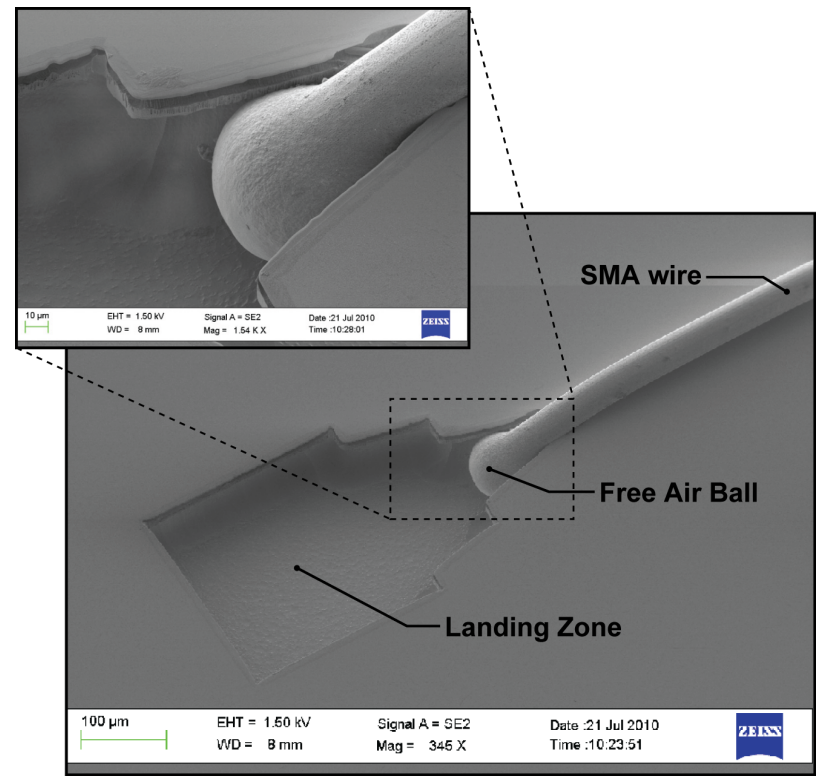

Figure 3: SEM image of an anchor structure, that was fabricated by combined anisotropic and isotropic deep reactive ion etching in three steps.

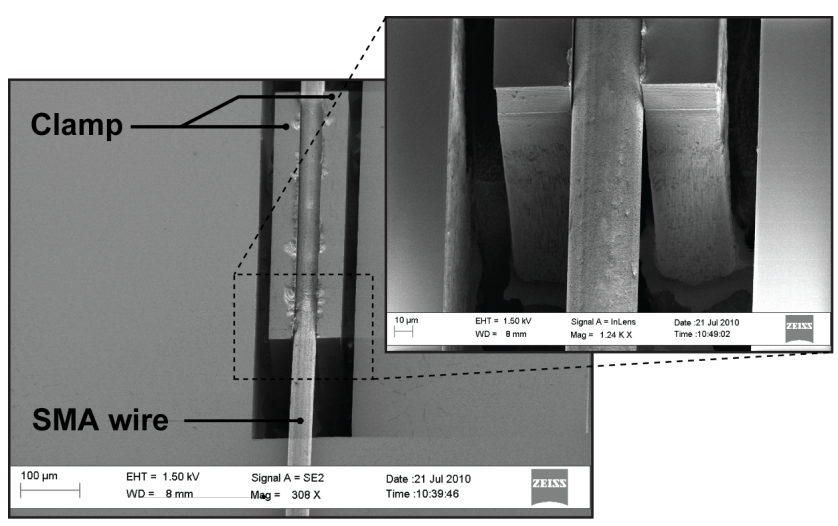

Figure 4: SEM image of a clamp fixation with a magnified view on one clamp feature. The wire is pushed in between the facing pair of cantilevers with the help of the wire bonder. Four similar clamps are used for the fixation of each wire.
Clamping structures with cantilever dimensions of $50 \times 500 \times 220 \mu \mathrm{m}$, as depicted in Fig. 4, generate a sufficiently high clamping force to fixate the SMA wire. A force of $1.2-1.3 \mathrm{~N}$, applied with the wire bonder, was necessary to snap the wire into the clamp structure.

The final step of the wire integration is the wire truncation, which was performed analog to a stitch bond. However, the use of a very high bond force of $1.2-1.3 \mathrm{mN}$ and ultrasonics result in a straight cut of the wire and, in contrast to conventional wire bonding, not in a deformation and micro weld of the wire to the surface, as depicted in Fig. 5. This indicates also that it is not feasible to bond SMA wires by the means of traditional wire bonding.

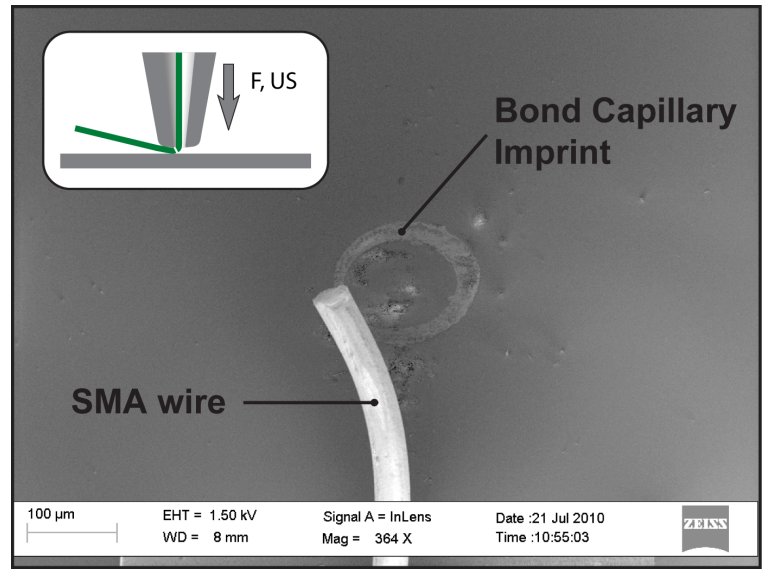

Figure 5: SEM image of a truncated SMA wire. The use of a high bond force and ultrasonics result in a straight cut of the wire and in contrast to conventional wire bonding not in a deformation and micro weld of the wire to the surface.

The presented wafer-level integration method also comprises the alignment of the SMA wire on chip-level. The placement accuracy of this integration method was analysed with an optical profilometer (Wyko NT9300). As depicted in Fig.6, both the in-plane wire placement deviation from the ideal geometrical anchor-to-clamp center line and the distance from the wire to the substrate (i.e. chip-level) was determined. The sample, a $75 \mathrm{~mm}$ long SMA wire with a diameter of $37.5 \mu \mathrm{m}$ was integrated on a $100 \mathrm{~mm}$ silicon substrate. The in-plane average deviation is $4.19 \pm 4.22(\sigma) \mu \mathrm{m}$ with a maximum of $13.9 \mu \mathrm{m}$. The out-of-plane distance of the SMA wire to the substrate is in average $15.6 \pm 4.5(\sigma) \mu \mathrm{m}$ and maximal $23.2 \mu \mathrm{m}$ (Fig. 6). This is an important measure for the design of the chip-level fixation of the SMA wire, which could e.g. be performed with Ni-electroplating in a subsequent step [9]. In addition, a basic evaluation of the mechanical robustness of the anchor and clamp structures was performed by implementing a simple actuator. This actuator is based on a $300 \mu \mathrm{m}$ thick silicon cantilever with a length of $100 \mathrm{~mm}$ and a width of $2 \mathrm{~mm}$ as cold state reset and two parallel integrated $75 \mathrm{~mm}$ long SMA wires as actuation. The energy input is provided by a hotplate on which the actuator is fixed on one side to 
enhance the thermal contact. Fig. 7a) illustrates the crosssection of the actuator both in actuated and idle position. Fig. 7b) depicts the actuator in a semi and Fig. 7c) in fully actuated state. It reveals that both SMA wire fixation elements withstand the force that is generated by the SMA wires, which is $200 \mathrm{mN}$ according to the wire data sheet [11]. The actuation was performed several hundred times without failure.

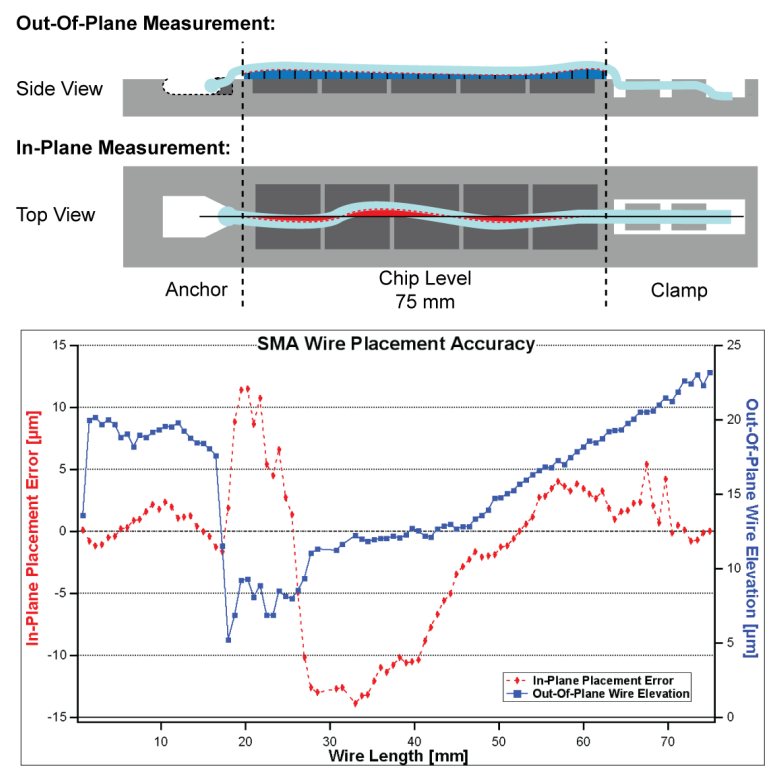

Figure 6: White Light Interferometric measurement data for the alignment accuracy of a SMA wire with a length of $75 \mathrm{~mm}$ and a diameter of $37.5 \mu \mathrm{m}$. As indicated in the drawing, the out-of-plane measurement (blue straight graph) determines the distance between the wire and the substrate, the in-plane measurement (red dashed graph) determines the lateral wire placement deviation from an ideal geometrical anchor-toclamp center line.

a)

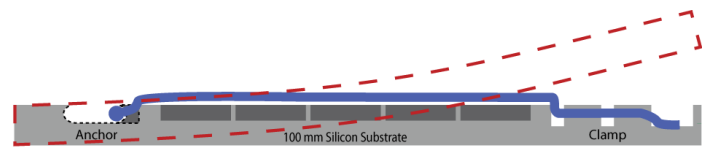

b)

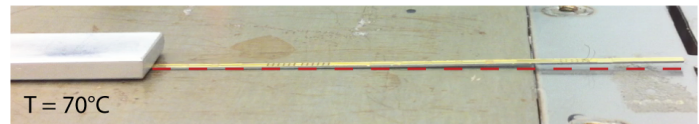

c)

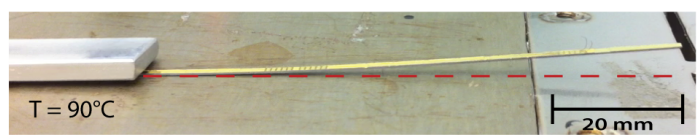

Figure 7: In order to verify the mechanical stabilty of the presented wire fixation approach, two $75 \mathrm{~mm}$ long SMA wires have been integrated in parallel on a silcon chip with a size of $3 \times 100 \mathrm{~mm}$. a) drawing of a cross-section, the red dashed outline represents the chip in a hot state. b) image of an slightly actuated device on a hotplate with a temperature of $70{ }^{\circ} \mathrm{C}$. c) device with increased actuation at $90{ }^{\circ} \mathrm{C}$.

\section{DISCUSSION AND CONCLUSIONS}

We have successfully demonstrated wafer level integration of shape memory alloy wires using standard wire bonder equipment. Even though the SMA wire integration was performed with a semi-automatic wire bonder an excellent placement accuracy could be achieved in this work. The usage of fully automated wire bonding equipment with optical pattern recognition and alignment systems potentially further improve these results and enables the implementation of this technique for mass production. The anchoring and clamping of SMA wire showed an excellent robustness.

\section{ACKNOWLEDGEMENTS}

The authors would like to thank Johnson Matthey Plc for supplying SMA wires and their support.

\section{REFERENCES}

[1] M. Kohl et al.: "Shape memory microactuators", Springer, pp. 23-24, (2004)

[2] K. Skrobanek et al.: "Stress-optimized shape memory microvalves", MEMS Proc., pp. 256-261, (1997)

[3] P. Krulevitch et al.: "Thin film shape memory alloy microactuators", J. Microelectromech. Syst., vol. 5, no. 4, pp. 270 - 82 (1996)

[4] S. Miyazaki et al.: "Development of high-speed microactuators utilizing sputterdeposited TiNi-base shape memory alloy thin films", Actuator Proc., pp.372-377 (2008)

[5] D. Clausi et al.: "Design and wafer-level fabrication of SMA wire microactuators on silicon", JMEMS, vol. 19 , no. 4 (2010)

[6] W.J.. Greig et al.: "Integrated circuit packaging, assembly and interconnections", Springer, pp.103-128, (2007)

[7] K. Gall et al.: "Instrumented micro-indentation of NiTi shape-memory alloys", Acta Materialia, vol. 49, no. 16, pp. 3205-3217 (2001)

[8] N. Roxhed et al.: "A method for tapered deep reactive ion etching using a modified Bosch process", JMM, vol. 17, pp. 1087-1092, (2007)

[9] D. Clausi et al.: "Wafer-level mechanical and electrical integration of SMA wires to silicon MEMS using electroplating", MEMS Proc. (2011)

[10] T. Goryczka et al.: "Characterization of nitrided/oxidized layers covering NiTi shape memory alloy", Solid State Phenomena, vol. 54, pp. 151-154, (2007)

[11] DYNALLOY, Inc.,"Technical characteristics of flexinol actuator wires", Datasheet, F1140Rev H, pp. 6 